\title{
CitH3: An Early Detection of Sepsis Following Hemorrhagic Shock? A View
}

\author{
Manoj Kumar*1, Sanjeev Bhoi ${ }^{2}$ and Sagar Galwankar ${ }^{3}$ \\ ${ }^{1}$ Department of Microbiology, Al-Falah School of Medical Science and Research Centre, India \\ ${ }^{2}$ Department of Emergency Medicine, JPNATC, AIIMS, India \\ ${ }^{3}$ Faculty of Emergency Medicine \& Global Health, University of South Florida, USA
}

Received: March 05, 2018; Published: March 20, 2018

*Corresponding author: Manoj Kumar, Tutor, Department of Microbiology, Al-Falah School of Medical Science and Research Centre, Dhauj, Faridabad, Haryana-121004, India, Tel: 9654965259; Email: manojaiims84@gmail.com

Abbrevations: HS: Hemorrhagic Shock; NETs: Neutrophil Extracellular Traps; CitH3: Citrullination of Histone H3; PTM: Post-Translational Modification; PAD: Peptidylarginine Deiminase; ETs: Immune Cells Extracellular Traps

\section{Commentary}

Hemorrhagic shock (HS) induces inflammatory cytokines leads to susceptible to infection, sepsis and multi-organ failure [1]. Sepsis is remaining leading cause of death within intensive care unit after trauma. Mortality rates are approximately 20-30\%. [2]. Ginsburg, Reported that the main cause of death in sepsis is the release from neutrophil nets of nuclear histone, highly toxic to endothelial cells and that these polycations are major and unique virulence factors Ginsburg et al. [3]. Neutrophils play an important role as the first line of defence of the immune system. In addition, one role of neutrophils, called neutrophil extracellular traps (NETs), has been discovered recently [3]. NETs are fibrous structures that are released extracellularly from activated neutrophils in response to infection and also the sterile inflammatory process. Modification by citrullination of histone H3 (CitH3) is thought to be involved in the in vitro formation of NETs $[4,5]$.

Recently, Li et al. [5] demonstrated that in a rodent model, lipopolysaccharide-induced shock, an increase in circulating Cit H3 is associated with sepsis.5 Citrullination of histones, in specific histone H3, was shown as a conjugation point for diverse inflammatory signals that trigger the neutrophil response to infections. Previous study reported that Cit H3 could be a potential serum biomarker for the early diagnosis of septic shock [6]. Moreover, Epigenetic process such as post-translational modification (PTM) of histones by acetylation are master regulators of gene expression and play a critical role in inflammatory and host defense responses. Citrullination of histones, another PTM catalyzed by peptidylarginine deiminase (PAD)-4 (PAD4), has recently been identified as an early step in a new type of cell death termed NETosis or ETosis, which is characterized by the release of neutrophil extracellular traps (NETs) or other immune cells' extracellular traps (ETs).

PAD4-mediated NET formation contributes to the mortality associated with shock/sepsis and may play a role in the pathobiology of end organ injury in response to combined hemorrhage plus sepsis. Lundberg et al. [5,6] demonstrated that citrullinated proteins in arthritic joints of experimental animals are correlated with severity of inflammation. However, these proteins associated with autoimmune diseases [7]. Previous study reported that Cit H3 alternative markers with higher sensitivity, specificity, and predictive value could help in the early detection and monitoring of sepsis progression, as well as the response to treatment $[3,5]$. However, poorly understood the role of Cit $\mathrm{H} 3$ in pathogenesis of sepsis in trauma hemorrhagic shock. Therefore, to explore the Cit3 H3 may be used as an alternative biomarkers that predict risk of developing sepsis in patient with T/HS and could be help in the management of subjects with sepsis. Need to be attention on this field.

\section{References}

1. Kumar M, Sharma K, Bhoi S, Kumar M, Pol MM (2016) Expression of p-38MAPK, GSK, JNK, ERK signaling: Can it be used as molecular markers among trauma hemorrhagic shock patients? J Emerg Med Trauma Shock 9: 131-132.

2. Mira JC, Szpila BE, Nacionales DC, Lopez MC, Gentile LF, et al. (2016) Patterns of gene expression among murine models of hemorrhagic shock/trauma and sepsis. Physiol Genomics 48(2): 135-144. 
3. Ginsburg I, Koren E, Varani J, Kohen R (2016) Nuclear histones: major virulence factors or just additional early sepsis markers? A comment. Inflammopharmacology 24(5): 287-289.

4. Pan B, Alam HB, Chong W, Mobley J, Liu B, et al. (2017) CitH3: a reliable blood biomarker for diagnosis and treatment of endotoxic shock. Sci Rep 7(1): 8972.

5. Li Y, Liu Z, Liu B, Zhao T, Chong W, et al. (2014) Citrullinated histone H3: novel target for the treatment of sepsis. Surgery 156(2): 229-234.
6. Biron BM, Chung CS, Chen Y, Wilson Z, Fallon EA, et al. (2018) PAD4 Deficiency Leads to Decreased Organ Dysfunction and Improved Survival in a Dual Insult Model of Hemorrhagic Shock and Sepsis. J Immunol 200(5): 1817-1828.

7. Lundberg K, Nijenhuis S, Vossenaar ER, Palmblad K, Van Venrooij WJ, et al. (2005) Citrullinated proteins have increased immunogenicity and arthritogenicity and their presence in arthritic joints correlates with disease severity. Arthritis Res Ther 7(3): R458-467.

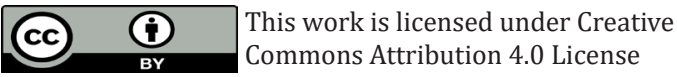

Submission Link: https://biomedres.us/submit-manuscript.php

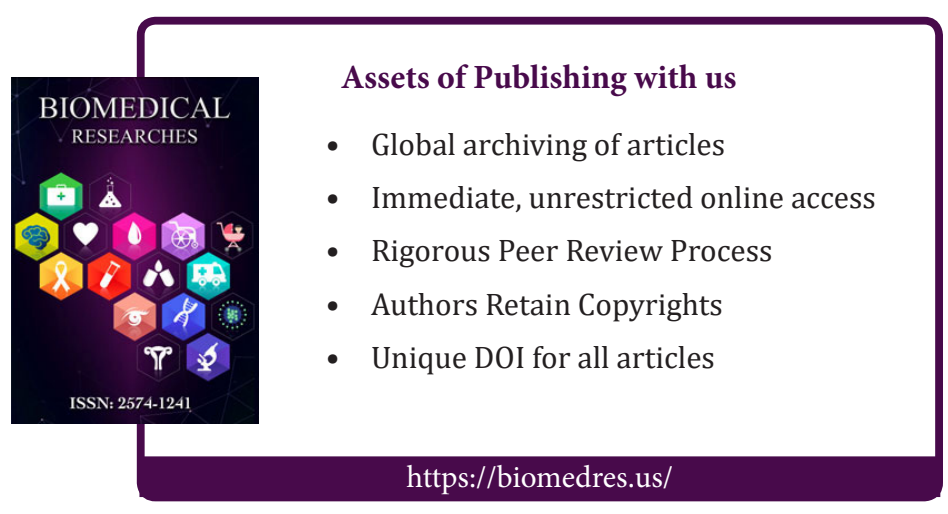

